6

\title{
State-of-the-art fluid management in the operating room
}

\author{
Timothy E. Miller, MB ChB, FRCA, Assistant Professor ${ }^{a, 1}$, \\ Karthik Raghunathan, MD MPH, Assistant Professor ${ }^{\text {a, b, }}$, \\ Tong J. Gan, MD, MHS, FRCA, Professor ${ }^{\text {a, * }}$ \\ a Department of Anesthesiology, Duke University Medical Center, Durham, NC 27710, USA \\ ${ }^{\mathrm{b}}$ Durham VAMC, Durham, NC 27710, USA
}

\section{Keywords:}

crystalloid

colloid

fluids

goal-directed fluid therapy
The underlying principles guiding fluid management in any setting are very simple: maintain central euvolemia, and avoid salt and water excess. However, these principles are frequently easier to state than to achieve. Evidence from recent literature suggests that avoidance of fluid excess is important, with excessive crystalloid use leading to perioperative weight gain and an increase in complications. A zero-balance approach aimed at avoiding fluid excess is recommended for all patients. For major surgery, there is a sizeable body of evidence that an individualized goal-directed fluid therapy (GDFT) improves outcomes. However, within an Enhanced Recovery program only a few studies have been published, yet so far GDFT has not achieved the same benefit. Balanced crystalloids are recommended for most patients. The use of colloids remains controversial; however, current evidence suggests they can be beneficial in intraoperative patients with objective evidence of hypovolemia.

(c) 2014 Elsevier Ltd. All rights reserved.

\footnotetext{
* Corresponding author. Tel.: +1 919681 4660; Fax: +1 9196814698.

E-mail addresses: timothy.miller2@duke.edu (T.E. Miller), Karthik.Raghunathan@duke.edu (K. Raghunathan), tjgan@duke. edu, tong.gan@duke.edu (T.J. Gan).

1 Tel.: +1919681 9660; Fax: +19196814698.

2 Tel.: +1919286 6938; Fax: +1919286 6853 .
} 
The underlying principles guiding fluid management in any setting are very simple: maintain central euvolemia, and avoid salt and water excess, in other words, maintain a full circulation to allow normal optimal cellular perfusion while avoiding any peripheral or interstitial edema, and associated increase in body weight. This, of course, is frequently easier to state than to achieve, particularly in the operating room (OR), where blood loss and fluid shifts can make fluid management challenging.

These principles have been displayed graphically as a U-shaped curve (Fig. 1) [1]. Episodes of hypovolemia, if undetected, can lead to hypoperfusion and organ dysfunction, with associated adverse outcomes [2]. Importantly, and perhaps not as readily acknowledged, excess fluid administration can result in tissue edema and adverse outcomes [3]. While both these processes can be extreme, more commonly, they are subtle with the splanchnic circulation particularly at risk.

One of the first responses to hypovolemia is recruitment of splanchnic reserves, potentially reducing flow in the splanchnic circulation to maintain core perfusion to vital organs. In healthy volunteers, normal core perfusion can be maintained after a controlled hemorrhage of $25 \%$ of blood volume, with no symptoms of hypovolemia and minimal change in heart rate or blood pressure [4]. However, at the same time, gastric tonometry shows evidence of splanchnic hypoperfusion once the circulating volume deficit exceeds $10 \%$ [5]. Other studies have also shown that the gut is the first area to suffer hypoperfusion as a result of acute hypovolemia $[5,6]$.

Conversely, excess fluid administration will result in fluid shifting out of the circulation and into the interstitium [7]. Again, the gut is at risk, and the resulting interstitial edema can result in edema of the gut wall and prolonged postoperative ileus. Even a modest positive salt and water balance causing a weight gain of $3 \mathrm{~kg}$ after elective colonic resection has been shown to be associated with delayed recovery of gastrointestinal function, increased complications, and extended hospital stay [8].

Gastrointestinal (GI) dysfunction is thus the most common perioperative complication after abdominal surgery [9]. Any delay in gastrointestinal recovery correlates closely with the duration of hospital stay, with discharge from the hospital frequently possible the day following GI recovery [10].

\section{Zero-balance approach to fluid therapy}

A number of studies in the literature have examined whether a "restrictive" fluid regimen is associated with fewer complications then a "liberal" fluid regimen. However, the terminology is confusing with widespread variation between studies in fluid regimens making interpretation difficult [11]. The term "restriction" is commonly interpreted to imply hypovolemia, whereas it may simply represent avoidance of the fluid excess seen in the "liberal" group.

One of the most well-known and frequently cited "restrictive" studies is the study by Brandstrup et al. published in 2003. This multicenter trial randomized 172 mainly ASA I-II patients to either a

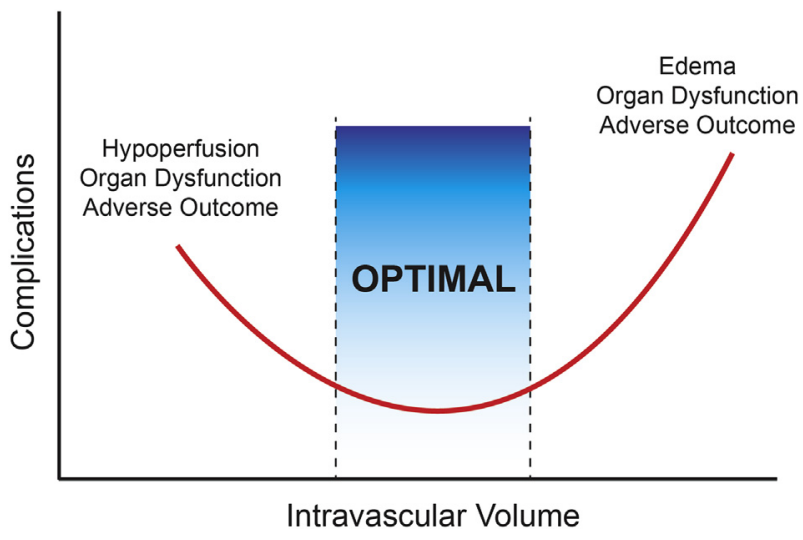

Fig. 1. Fluid load versus complications (modified from Bellamy) [1]. Both hypovolemia and hypervolemia can lead to organ dysfunction and adverse outcomes. The challenge is to keep patients at all times in the optimal zone. 
"restricted" or a standard intraoperative and postoperative intravenous (IV) fluid regimen. The restricted regimen aimed to maintain preoperative body weight, whereas the standard regimen resembled everyday practice, including the replacement of "third-space" fluid loss. The results showed that the "restrictive" group significantly reduced the incidence of major and minor complications, such as anastomotic leakage, pulmonary edema, pneumonia, and wound infection. However, a closer look at the fluid protocols reveals that in the "restrictive" group patients received a mean of $2740 \mathrm{ml}$ of fluid on the day of surgery, which was a mixture of crystalloids and colloids. By contrast, the liberal group received over $5388 \mathrm{ml}$ of fluid with the majority of the extra fluid being normal saline (NS), with four patients needing re-intubation for pulmonary edema. A common interpretation of this study is that the liberal group received too much fluid and the "restrictive" group had appropriate fluids. Indeed, the authors found a dose-response relation between complications, increasing volumes of IV fluid $(P<0.001)$, and increasing body weight $(P<0.001)$ on the day of operation independent of the allocation group.

Other restrictive studies are varied in terms of design, outcome variables, types of fluid administered, and definition of fluid restriction. Indeed, a restrictive regimen in one study may be liberal in another. Brandstrup et al. have recently called for the term restrictive to be abandoned as it has caused so much confusion and replaced by zero balance, and we support this view [12].

Therefore, an important question is why the liberal administration of fluid was commonplace until recently to replace presumed volume deficits such as third-space and evaporative losses frequently resulting in an increase of 3-6 kg in body weight perioperatively. The third-space theory was developed in the 1960s, being described as a nonfunctional compartment where fluid could be sequestered, and therefore needed filling [13]. Recent evidence does not support the existence of a third-space [7]. Fluid is simply either in the intravascular space or in the interstitium. Administering excess fluid to induce a state of hypervolemia will increase the hydrostatic pressure within the circulation with subsequent atrial natriuretic peptide (ANP) release, damage to the glycocalyx, and increased leak of fluid from the circulation into the interstitium as eloquently described by Chappell et al. [7] This "third spacing" is then frequently replaced with more IV fluid creating a cycle in which fluid accumulates within the interstitium and the patient gains weight over the perioperative course. Empiric filling of this presumed "space" is not warranted, and we believe that the term "third-space" should be abandoned. Similarly, perioperative evaporative losses have also been exaggerated [14]. It is now more than 30 years since Lamke et al. elegantly demonstrated by direct measurement of the basal evaporation rate that typical fluid losses during major abdominal surgery are $0.5-1 \mathrm{ml} / \mathrm{kg} / \mathrm{h}$ [15].

Our first goal for perioperative fluid therapy should therefore be the avoidance of excess fluid. As crystalloids are routinely used for the maintenance of hydration, avoiding excess crystalloids is critical. A zero-balance fluid approach may be achieved with a background infusion of a balanced crystalloid at $2-3 \mathrm{ml} / \mathrm{kg} / \mathrm{h}$ based on ideal body weight. For many operations, adopting a zero-balance approach may be sufficient. However, during major surgery, there is also a significant body of evidence supporting the goal-directed fluid therapy (GDFT) approach aimed at individualizing fluid therapy.

\section{Individualized GDFT}

Goal-directed therapy (GDT) is a term that has been used for nearly 30 years to describe methods of optimizing fluid and hemodynamic status to improve outcome for high-risk surgical patients. However, the terminology used in the literature is inconsistent and confusing [16]. The term was first used to describe early oxygen-targeted GDT in the 1980s and the 1990s that used the pulmonary artery catheter (PAC) to augment oxygen delivery to supranormal levels in high-risk surgical patients. More recently, a number of minimally invasive monitors of flow-based hemodynamic parameters have been developed. GDFT refers to individualized fluid management with or without inotropes guided by cardiovascular measures of fluid responsiveness by preemptively maximizing stroke volume (SV) while also avoiding fluid overload. Fluids are given only if there is demonstrable fluid responsiveness and otherwise are withheld. This technique has also been referred to in the literature as SV optimization [17]; for the purposes of this article, we will use the term GDFT.

In theory, GDFT enables an objective assessment of each patient's individual cardiovascular response to the bolus administration of IV fluids. Most of these monitors use methods of predicting 
fluid responsiveness such as stroke volume variation (SVV), pulse pressure variation (PPV), corrected flow time (FTc), or pleth variability index (PVI). This is an increasingly competitive market with a growing number of noninvasive devices available for the clinician.

Two important questions regarding individualized fluid therapy are as follows:

1. Can we measure the response to a fluid challenge?

2. Can we predict if a patient is going to respond to a fluid challenge?

How can we measure the response to a fluid challenge?

Minimally invasive flow-based monitors are able to estimate the cardiac output (CO) and SV that can then be used to monitor the response to a fluid challenge (Fig. 2). This provides a sophisticated way of titrating IV fluid for complex patients. GDFT utilizes algorithms incorporating fluid challenges to optimize SV and avoid episodes of hypovolemia and postoperative oxygen debt.

Can we predict if the patient is going to be fluid responsive?

Arterial waveform analysis with a minimally invasive CO monitor also measures dynamic parameters of fluid responsiveness, based on cardiopulmonary interactions, such as SVV and PPV. These dynamic variables are superior to traditional static indices such as CVP and are reliable indices of volume responsiveness in mechanically ventilated patients with consistent and sufficient cardiorespiratory interaction (adequate tidal volumes in sinus rhythm and closed chest conditions).

The physiology behind SVV and PPV is: positive pressure ventilation induces cyclical changes in the loading conditions of the right ventricle (RV), with a reduction in preload during mechanical insufflation (the inspiration phase) [18]. This leads to cyclical changes in RV SV corresponding to the respiratory cycle, which is at a minimum at the end of the inspiratory period. After the pulmonary transit time (a lag period of two to three beats for the blood to travel through the pulmonary system), left ventricle (LV) preload reduction will occur, resulting in a reduction in LV SV that is minimum at the end of the expiratory period.

In volume-depleted patients, when the ventricle is operating on the steep part of the Starling curve, the magnitude of these changes in SV and pulse pressure will be greater. This will manifest itself as a characteristic systolic "swing" in the arterial line pressure waveforms with respiration. PPV and SVV

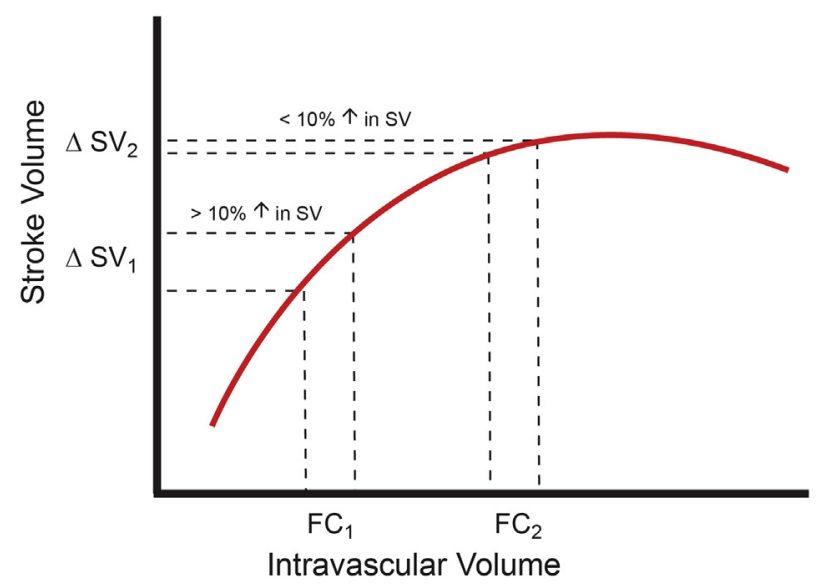

Fig. 2. Frank-Starling-based stroke volume optimization. When a patient is hypovolemic and on the steeper ascending part of the Frank-Starling curve, an intravenous fluid challenge $\left(\mathrm{VC}_{1}\right)$ will lead to a $>10 \%$ increase in SV. Such a patient has "recruitable" SV, and is in a fluid-responsive state. When the patient is no longer hypovolemic $\left(\mathrm{VC}_{2}\right)$, the same fluid challenge will result in a $<10 \%$ increase in stroke volume. The patient is now not fluid responsive and will not benefit from a further fluid challenge. 
represent "virtual" preload challenges occurring during each respiratory cycle in ventilated patients. There is no need to administer fluid to predict respondents, with an SVV or PPV $>12-13 \%$ accurately predicting a positive response to a fluid challenge [19-21]. As these dynamic indices predict respondents with more accuracy than CVP and as no CVP threshold value is reliable to diagnose fluid responsiveness, there is less need for invasive lines merely for the purpose of monitoring [19,22]. In several studies comparing PPV and SVV, PPV appears to be the most sensitive and specific variable $[19,20,23]$.

The use of dynamic parameters to assess fluid responsiveness does have some important limitations. PPV and SVV require a constant R-R interval (i.e., normal sinus rhythm) and "adequate" pressures in the chest and abdomen, otherwise their predictive value is decreased [24]. The technique relies on cardiopulmonary interactions, and therefore any change in tidal volume and/or intrathoracic pressure will affect the interaction. Raised intra-abdominal pressure will cause higher intrathoracic pressures and an exaggerated cardiopulmonary interaction that will raise the threshold for volume responsiveness independent of preload [23]. Only PPV has been validated in this situation, and the clinician needs to understand the physiology and look for the response to a fluid challenge to individualize the threshold for fluid responsiveness [23]. For example, with a pneumoperitoneum, a patient may have a PPV of $15 \%$ when euvolemic, and only respond to a fluid challenge with a $10 \%$ increase in SV when PPV > 20\%. This raised threshold can then be used to predict when further fluid challenges are needed.

\section{Does GDFT improve outcomes?}

There are a number of technologies that can be used for GDFT. The most widely studied is the esophageal Doppler monitor (EDM, Deltex Medical, Chichester, UK). The Doppler probe is placed in the esophagus and focused at the descending thoracic aorta, where it uses the Doppler principle to measure blood flow velocity and produce a waveform for velocity versus time. This velocity-time integral (area under the velocity vs. time curve) is then converted to SV using a nomogram of height, weight, and age to estimate the cross-sectional area of the descending aorta. EDM-derived SV measurements have been well validated in different environments and clinical scenarios.

There are a number of studies that show improved outcomes with EDM-guided fluid optimization, as demonstrated by a faster return in gastrointestinal function, a reduction in postoperative complications, and reduced length of stay (LOS) [25]. All of these studies used an algorithm to optimize SV (serial fluid boluses when a $>10 \%$ improvement in SV is present), often combined with assessment of FTc to predict fluid responsiveness; and all used background crystalloid infusions with colloid boluses for optimization.

Mythen and Webb demonstrated that GDFT reduced the incidence of gastrointestinal mucosal hypoperfusion and major complications after cardiac surgery [6]. Gan et al. randomized 100 major elective noncardiac surgical patients with an expected blood loss $>500 \mathrm{ml}$ to either routine care or EDM-guided GDFT [26]. Patients in the GDFT group had significantly shorter median LOS (5 vs. 7 days, $p<0.05$ ) and an earlier ability to tolerate solid food ( 3 vs. 5 days, $p<0.05$ ). The studies by Noblett [27] and Wakeling [28] showed a similar reduction in LOS in colorectal surgical patients, as well as a shorter time to full oral diet suggesting improved gastrointestinal function.

Although the EDM has the most evidence of an outcome benefit, some experience is required to become skilled in using this device, and an optimal signal may be difficult to obtain. Frequent readjustment may often be necessary to maintain alignment with flow. In addition, there is some concern that the device only measures flow through the descending aorta and that some of the mathematical assumptions may make it somewhat inaccurate [29], and the use of electrocautery can interfere with the signal.

There are a number of $\mathrm{CO}$ monitors that utilize pulse contour analysis of the arterial waveform (e.g., LiDCO, Vigileo, and PiCCO) to calculate SV, CO, as well as dynamic measures of preload responsiveness, such as PPV and SVV. There are an increasing number of positive outcome studies using these devices. The PiCCO and LiDCOplus systems use transpulmonary thermodilution and lithium, respectively, to calibrate the SV obtained from pulse pressure analysis. These systems are therefore suitable for use in high-risk surgery or the intensive care unit (ICU). Goal-directed intraoperative hemodynamic 
optimization using the PiCCO has been shown to decrease requirement for inotropes/vasopressors and shorten mechanical ventilation time in patients undergoing routine cardiac surgery [30]. Pearse et al. used the LiDCOplus system (LiDCO Ltd, Cambridge, UK) to optimize high-risk surgical patients postoperatively, using a combination of colloid and inotropes [31]. There were fewer complications (44\% vs. $68 \%$ ) and a shorter LOS in the GDT group.

By contrast, the Flotrac/Vigileo (Edwards, Irving, TX, USA) and LiDCOrapid (LiDCO Ltd, Cambridge, UK) systems do not require calibration and are more commonly used for GDFT. Benes et al. randomized 120 patients undergoing major abdominal surgery with expected blood loss $>1000 \mathrm{ml}$ to either GDFT with the Flotrac/Vigileo or routine intraoperative care [32]. The aim of the GDFT group was to maintain $\mathrm{SVV}<10 \%$ using colloid boluses of $3 \mathrm{ml} / \mathrm{kg}$. Results showed better intraoperative hemodynamic stability, decrease in serum lactate at the end of surgery, and a lower incidence of postoperative organ complications ( $34 \%$ vs. $77 \% ; p=0.0066$ ) with GDFT.

GDFT using the Flotrac/Vigileo has also been shown to improve outcomes in various different patient groups. Cecconi et al. showed improved outcomes in 40 patients receiving regional anesthesia for hip arthroplasty [33]. Kapoor et al. showed a decrease in the duration of mechanical ventilation, ICU LOS, and hospital LOS in patients undergoing cardiac surgery [34]. Targeting a PPV $<10 \%$ has been shown to improve postoperative outcomes and reduce length of intensive care and hospital stay in high-risk surgical patients [35].

However, clinical medicine is rarely "never or always" where patients with a PPV $<10 \%$ will never respond to fluids, and patients with a PPV $>10 \%$ will always respond to fluids. Therefore, a "gray zone" approach has been advocated with two cutoffs: patients with a PPV $<9 \%$ exclude fluid response with near certainty, whereas patients with a PPV $>13 \%$ will nearly always respond to fluids (Table 1) [36]. Approximately $25 \%$ of patients are in the "gray zone" (PPV 9-13\%) where PPV is inconclusive: therefore, the decision to give fluids or not will depend on the overall picture, trends, and the individual needs of the patient (i.e., if the patient has renal dysfunction, it may be prudent to keep the PPV $<9 \%$, whereas if the patient has pulmonary dysfunction avoiding fluid excess may be a higher priority). A smaller fluid challenge could also be considered [37]. This approach seems sensible and practical.

Other noninvasive technologies that can be used for GDFT include the noninvasive CO monitor (NICOM, Cheetah Medical, Vancouver, WA, USA) and the Nexfin (Edwards, Irving, TX, USA). The NICOM is a bioreactance-based monitor that has been shown to perform similarly to the ED in guiding GDFT [38]. The Nexfin accurately and continuously measures both arterial blood pressure [39] and CO [40,41] using an inflatable finger cuff.

Hamilton et al. performed a systematic review and meta-analysis recently on the use of GDT therapy in moderate- to high-risk surgical patients [42]. Twenty-nine studies were identified involving 4805 patients that used various forms of hemodynamic monitoring including the PAC, esophageal Doppler, LiDCO, PiCCO, and FloTrac. Interventions consisted of fluid therapy with or without inotropic support. The authors found that the use of preemptive hemodynamic monitoring significantly reduced surgical complications (odds ratio $0.43(0.34-0.53) ; P<0.0001$ ). Subgroup analysis revealed that this effect was similar regardless of the type of monitor used and the type of intervention.

The Center for Medicare Services in the USA reviewed the literature and supported a professional fee being paid to clinicians using EDM-guided perioperative volume optimization [43]. The EDM was endorsed by the "National Institute for Health and Care Excellence" (NICE) in the UK in 2011. They stated "the case for adopting the Esophageal Doppler in the NHS is supported by the evidence; there is a reduction in in-hospital complications, use of central venous catheters, and LOS compared with conventional clinical assessment with or without invasive cardiovascular monitoring. The esophageal

Table 1

Frequency and number of respondents to a fluid challenge at different PPV values [36].

\begin{tabular}{lrc}
\hline & Patients (\%) & Respondents (\%) \\
\hline PPV $<9 \%$ & $159(38 \%)$ & $18(11 \%)$ \\
PPV 9-13\% & $98(24 \%)$ & $49(50 \%)$ \\
PPV $>13 \%$ & $156(38 \%)$ & $142(91 \%)$ \\
\hline
\end{tabular}

PPV $=$ Pulse Pressure Variation. 
Doppler should be considered for use in patients undergoing major or high-risk surgery." [44] Many clinicians have expanded this recommendation to other technologies that can be used for GDFT.

\section{Recent studies of GDFT within an Enhanced Recovery program}

In the past few years, a number of studies have examined the use of the GDFT with the EDM within Enhanced Recovery After Surgery (ERAS) programs. ERAS programs comprise a range of therapeutic options designed to minimize the stress response to surgery and improve recovery (Table 2).

ERAS programs are becoming the standard of care for colorectal surgery, and are increasingly being applied to other surgeries. A meta-analysis has showed that ERAS programs reduced LOS for colorectal surgery by 2.5 days and decrease perioperative complications by $50 \%$ [45].

One of the principal aims of the preoperative component of an ERAS program is to "bring the patient to the OR in a fed state." Avoiding routine bowel preparation, allowing clear fluids until 2 h preoperatively, and drinking a preoperative carbohydrate drink can achieve a reduction in thirst, hunger, anxiety, and postoperative insulin resistance [46-48]. Patients compliant with these preoperative components are more likely to be euvolemic upon arrival to the OR, potentially making fluid management in the OR easier.

Studies of GDFT within an ERAS program have failed to find the same benefit on postoperative outcomes as the early studies. This is perhaps not surprising, as care within the control group has significantly improved making it harder to observe a difference versus GDFT. Srinivasa et al. randomized 85 patients to GDFT or a zero-balance fluid regimen within an ERAS program for colorectal surgery [49]. Total fluid given in both groups intraoperatively was statistically higher in the GDFT group (1994 vs. $1614 \mathrm{ml} ; p=0.010$ ), but at a glance the volumes are comparable, and much lower overall than previous GDFT studies outside of an ERAS program. Indeed, the GDFT protocol used was very similar to the protocol used in the Gan et al. study in 2002 when the total fluid given intraoperatively was $5420 \mathrm{ml}$ in the GDFT group and $4775 \mathrm{ml}$ in the control group [26]. The population studied by Gan was slightly different in that it included all abdominal surgery, but the difference in fluid requirements aptly illustrates the change in practice that has occurred over the last 10 years, with the trend towards less fluid use intraoperatively. Most patients were fluid responsive after induction in the Gan study and the use of GDFT significantly improved SV from baseline to the end of surgery. In comparison, in the Srinivasa study, patients were not fluid responsive; SV did not change from the beginning to the end of surgery, and was the same in both groups.

Brandstrup also found no benefit with GDFT compared to a zero-balance regimen within an Enhanced Recovery program [12]. Importantly, in both the Srinivasa and Brandstrup studies, GDFT was not associated with harm when compared with a zero-balance strategy [12,49]. By contrast, the multicenter OPTIMISE study focused on GDFT on high-risk patients undergoing major abdominal surgery [50]. A significant proportion of these patients were managed within an ERAS pathway. In this high-risk population, there was a nonsignificant trend towards decreased complications (36.6\% vs. $43.4 \%, p=0.07)$ and 180 -day mortality ( $7.7 \%$ vs. $11.6 \%, p=0.08)$ in the GDFT group compared to usual care.

Therefore, while ERAS programs may have raised the threshold for benefit, there will still be many patients, whether expected or unexpected, for whom SV optimization will be beneficial. This is the same as any other monitoring device; not all patients' care will benefit pulse oximetry, but it is rightly

Table 2

Duke ERAS program for colorectal surgery [77].

\begin{tabular}{lll}
\hline Preoperative & Intraoperative & Postoperative \\
\hline Identify patients & Thoracic epidural & Early feeding \\
Educate about program & Goal-directed fluid therapy & Early mobilization \\
Clear fluids until $2 \mathrm{~h}$ before surgery & Multimodal Analgesia & Optimize fluid regimen \\
Carbohydrate drink & Antibiotics before incision & Optimize analgesic regimen \\
Selective bowel preparation & PONV and thromboprophylaxis & No nasogastric tube nor urinary catheter \\
\hline
\end{tabular}

ERAS = Enhanced Recovery After Surgery; PONV=Postoperative Nausea and Vomiting. 
standard of care. Many patients who are undergoing major surgery outside of ERAS pathways (i.e., vascular surgery) will also benefit for GDFT. Guidelines have been published recommending the types of cases when GDFT should be considered [51]. Ultimately, the need for GDFT is patient, surgeon, procedure, and institution specific. Each institution needs to look at its outcomes and benchmark data for the benefitting population against other institutions before making an informed decision about appropriate implementation of GDFT. These data should include LOS, readmission rate, mortality, and costs. All patients should have an individualized plan for fluid management rather than a formulaic approach based on body weight and duration of surgical exposure. For patients undergoing colorectal surgery with limited blood loss within an ERAS pathway, minimal fluid may be required, and GDFT may not offer additional benefits [17]. However, current evidence suggests that for the majority of patients undergoing major surgery, GDFT remains the technique of choice for perioperative fluid and hemodynamic optimization [52].

\section{Which fluid should one use?}

An electrolyte-balanced crystalloid such as Lactated Ringers (LR), Plasma-Lyte ${ }^{\mathrm{TM}}$, or Normosol ${ }^{\mathrm{TM}}$ should be used to meet maintenance requirements. The recommended rate for a background infusion to replace insensible losses and urine output is in the range 1-3 ml $/ \mathrm{kg} / \mathrm{h}$ based on lean body weight [14]. However, $0.9 \%$ NS is still the most commonly used crystalloid in the world [53]. NS is not "normal" as it contains supraphysiologic levels of sodium and chloride (NS contains $154 \mathrm{mmol} / \mathrm{l}$ of chloride, which is significantly more than the plasma concentration of $105 \mathrm{mmol} / \mathrm{l})$. Administration of NS, or solutions formulated in NS, such as colloids suspended in saline, causes a predictable hyperchloremic metabolic acidosis, which is associated with harm [54-59]. In a recently published large retrospective database analysis comparing the administration of either NS alone or a balanced crystalloid solution (Plasma-Lyte ${ }^{\mathrm{TM}}$, Baxter, IL, USA) alone on the day of surgery, there was an increased use of special investigations (arterial blood gases and lactate levels) and treatments (buffers and blood products) in the NS group, presumably to investigate and manage saline-induced acid-base abnormalities [60]. When compared with a balanced electrolyte solution, the administration of NS also caused a significantly greater risk of postoperative infection and renal failure requiring dialysis.

It is known that hyperchloremia may affect renal function adversely. In denervated dog kidneys, intrarenal infusion of chloride-containing solutions produced renal vasoconstriction and a fall in the glomerular filtration rate [61]. Recently, Chowdhury et al. demonstrated the same effect in humans by measuring renal cortical perfusion during and following the infusion of 21 of saline compared with Plasma-Lyte ${ }^{\mathrm{TM}}$ [62]. In the ICU, the implementation of a chloride-restrictive fluid strategy was associated with a significant decrease in the incidence of acute kidney injury (AKI) and use of RRT [63]. In the OR, patients undergoing primary hip replacement had a significantly worse base deficit when randomized to NS compared to a balanced fluid regimen [64].

One area of practice where use of NS may be prudent is in traumatic brain injury. A post hoc subset analysis of the SAFE study for patients with traumatic brain injury $(n=460)$ revealed a lower mortality rate in patients treated with NS compared with albumin (33.2\% vs. 20.4\%) [65]. It is important to note that the albumin preparation used in the SAFE study, $4 \%$ Albumex $^{\circledR}$ (CSL, Melbourne), was relatively hypotonic (260 mOsmol/l), and this hypotonicity may have contributed to worse outcomes in the albumin group [66]. Otherwise, the use of balanced crystalloid solutions seems to be beneficial. Unfortunately, there have been no significant studies comparing different balanced regimens. In particular, there are no data comparing the different anions (lactate, acetate, and gluconate) used as an alternative to chloride.

Most studies of GDFT have used colloid boluses, predominantly starch solutions suspended in saline or balanced crystalloids, to optimize SV in addition to a maintenance crystalloid regimen. This is based on Starling's principle that a colloid solution containing macromolecules will remain in the intravascular compartment. By contrast, when the crystalloid is administered, only $20 \%$ of the solution will remain in the vascular compartment after $30 \mathrm{~min}$ [67]. Therefore, when the vascular barrier is intact perioperatively, it seems prudent according to physiologic principles to replace blood loss and plasma loss out of the circulation with boluses of an iso-oncotic colloid [14]. This is typically achieved with a 200-250-ml bolus. 
Recently, however, the use of colloids in medical practice, particularly starch solutions, has been questioned. Two large trials (CHEST [68] and 6S [69]) examining the use of hydroxyethyl starch (HES) in the ICU have shown that HES is associated with harm. When the SAFE study examining albumin use in the ICU is added to this list, there are now three large trials with over 15,000 patients showing no benefit from the use of the more expensive colloids solutions in the ICU. This has led many experts to question the use of colloid solutions perioperatively.

Although CHEST and 6S were well-conducted trials, it is important to note that a randomized controlled trial (RCT) is designed to answer a particular question in a particular setting. In $6 \mathrm{~S}$, for instance, which examined the use of HES in septic patients in the ICU, over $75 \%$ of patients in both groups received colloidal solutions before enrollment. This delayed enrollment may be due to the difficulty in enrolling patients into clinical ICU studies. Therefore, it can be argued the trial examined the use of colloids in a conventional manner (HES and other colloids for initial resuscitation, and then crystalloid during the stabilization phase of critical illness - crystalloid group) versus an unusual manner (exclusively HES during the post-resuscitation stabilization period). 6S answers an important question: HES should clearly not be used exclusively during the stabilization phase of critical illness, especially in sepsis. The applicability of this result to other settings is open to interpretation. The use of post-resuscitation fluid boluses of any kind in sepsis has limited success and may be harmful. Bihari et al. studied 184 fluid boluses in 50 patients with sepsis after initial resuscitation and found minimal increase in MAP and urine output following a fluid bolus in this context, yet a significant decrease in the $\mathrm{PaO}_{2} / \mathrm{FiO}_{2}$ ratio [70].

CHEST examined the use of HES or NS in 7000 patients admitted to ICU and showed an increase in renal replacement therapy (RRT) with the use of HES. However, proponents of HES might analyze the trial differently. Again, patients were generally resuscitated before enrollment, with the average time of enrollment $10 \mathrm{~h}$ after admission to ICU. The risk-adjusted $p$ value for the increase in RRT with HES was 0.05. Therefore, it could be concluded that in a large study of critically ill patients given HES at the wrong time (after resuscitation), there was a borderline increase in RRT in the HES group and no increase in mortality. To adequately power a trial to investigate any possible intraoperative harm from HES would need an exceptionally large trial and is unlikely to ever occur.

By contrast, the recent publication of the Colloids Versus Crystalloids for the Resuscitation of the Critically Ill (CRISTAL) trial, an open-label (clinicians were not blinded to the study fluid) randomized comparison of crystalloids against colloids early during the resuscitation phase of critical illness (i.e., among hypovolemic patients), has suggested a possible benefit for colloids [78]. Similarly, in an animal model, when hemorrhagic shock was induced by the removal of sufficient quantities of blood to obtain and sustain a mean arterial pressure (MAP) of $40 \mathrm{mmHg}$, restoration of MAP was four times faster with HES than LR $(p<0.001)$ and required fourfold less volume $(p=0.04)$ [71].

What inferences can we make from these trials for the perioperative use of HES and colloids in general? Context is probably key: a colloid only behaves like a colloid when the glycocalyx is intact and the patient is in need of intravascular expansion. The benefit of a colloid in the setting of an intact glycocalyx in the patient undergoing volume optimization during major surgery or after major hemorrhage may be very different from the risk of HES during the stabilization phase of severe sepsis with glycocalyx shedding and disruption.

Yates et al. recently conducted an RCT comparing the use of boluses of crystalloid or colloid for GDFT during major surgery [72]. They found no difference in outcomes although, similar to a previous study, $60 \%$ more fluid was needed in the crystalloid group [73]. Therefore, in clinical practice without significant hypovolemia, the colloid-to-crystalloid ratio appears to be approximately $1: 1.5[72,73]$. However, the volume effect of colloids is context sensitive [14], and when hypovolemia is present, the colloid-to-crystalloid ratio may be higher [71].

Importantly, in the Yates study, there was also a 38\% failure rate in the crystalloid group when a colloid bolus was given to achieve the hemodynamic targets. Blood loss in both groups was also low. From these results, it does seem that a crystalloid may be used as an alternative to colloid for SV optimization during GDFT. However, in the setting of acute blood loss, it may still be prudent to use a colloid and avoid crystalloid overload. 


\section{What about minor surgery?}

In patients undergoing minor or ambulatory surgery, a relatively generous crystalloid regimen has been shown to improve outcomes such as pain, nausea, and dizziness, and facilitate earlier discharge [74-76]. Although these studies were performed in the era when prolonged fasting was still the norm, there may be limited harm in infusing 1.5-2 1 of balanced crystalloid in most of these patients and possible benefit. Most patients undergoing ambulatory surgery will not have a postoperative IV infusion and can sometimes have reduced oral intake of fluids for $24 \mathrm{~h}$ perioperatively.

\section{Conclusion}

Perioperative fluid management is important. There is an increasing body of literature suggesting that both hypovolemia and fluid excess are associated with harm. All patients should therefore have an individualized plan for fluid management and appropriate hemodynamic monitoring. GDFT should be considered based on surgical complexity and patient risk factors.

\section{Practice points}

- Every patient undergoing surgery should have an individualized fluid management plan.

- A zero-balance approach aimed at avoiding perioperative weight gain is appropriate for most patients. Avoid boluses and use a low-volume infusion of balanced crystalloids.

- For patients undergoing major surgery, individualized GDFT using a minimally invasive cardiac output monitor is recommended.

- Crystalloids alone may be appropriate in situations where the glycocalyx is impaired (e.g., septic patients in the operating room).

- Colloids may be appropriate when patients are likely to have an intact glycocalyx and are receiving GDFT (e.g., in preemptive optimization for moderate or high-risk operations as this will avoid the use of larger volumes of crystalloids), and/or show evidence of objective hypovolemia.

- It is unclear whether synthetic colloids like starch solutions are appropriate - more data are needed.

\section{Research agenda}

- We need a large multicenter, double-blind RCT of colloids versus crystalloids in elective major surgery where fluids are given to treat objective evidence of hypovolemia. As ERAS is becoming a standard of care, this should be performed within an ERAS protocol.

- Further work is needed to determine when GDFT is useful in improving outcomes within an ERAS protocol.

- NS is still the most commonly used IV fluid around the world. Evidence is increasing that NS is associated with harm; however, practice change is difficult to achieve. A large, multicenter trial of balanced versus unbalanced fluid would help determine if the routine use of NS is justifiable. 


\title{
Conflict of interest statement
}

\author{
Miller: Honoraria - Edwards Lifesciences. \\ Raghunathan: Research Support - Baxter. \\ Gan: Honoraria - Baxter, Edwards Lifesciences, Hospira, Merck. Research support - Covidien, \\ Fresenius, Merck, Pacira, and Premier.
}

\section{References}

[1] Bellamy MC. Wet, dry or something else? Br J Anaesth 2006;97(6):755-7 [Epub 2006/11/14].

[2] Mythen MG, Webb AR. The role of gut mucosal hypoperfusion in the pathogenesis of post-operative organ dysfunction. Intensive Care Med 1994;20(3):203-9 [Epub 1994/01/01].

*[3] Brandstrup B, Tonnesen H, Beier-Holgersen R, et al. Effects of intravenous fluid restriction on postoperative complications: comparison of two perioperative fluid regimens: a randomized assessor-blinded multicenter trial. Ann Surg 2003; 238(5):641-8 [Epub 2003/10/28].

[4] Hamilton-Davies C, Mythen MG, Salmon JB, et al. Comparison of commonly used clinical indicators of hypovolaemia with gastrointestinal tonometry. Intensive Care Med 1997;23(3):276-81 [Epub 1997/03/01].

[5] Mythen MG, Webb AR. Intra-operative gut mucosal hypoperfusion is associated with increased post-operative complications and cost. Intensive Care Med 1994;20(2):99-104 [Epub 1994/01/01].

[6] Mythen MG, Webb AR. Perioperative plasma volume expansion reduces the incidence of gut mucosal hypoperfusion during cardiac surgery. Arch Surg 1995;130(4):423-9 [Epub 1995/04/01].

[7] Jacob M, Chappell D, Rehm M. The 'third space'-fact or fiction? Best Pract Res Clin Anaesthesiol 2009;23(2):145-57 [Epub 2009/08/06].

[8] Lobo DN, Bostock KA, Neal KR, et al. Effect of salt and water balance on recovery of gastrointestinal function after elective colonic resection: a randomised controlled trial. Lancet 2002;359(9320):1812-8 [Epub 2002/06/05].

[9] Bennett-Guerrero E, Welsby I, Dunn T], et al. The use of a postoperative morbidity survey to evaluate patients with prolonged hospitalization after routine, moderate-risk, elective surgery. Anesth Analg 1999;89(2):514-9 [Epub 1999/08/ 10].

[10] Delaney CP, Senagore AJ, Viscusi ER, et al. Postoperative upper and lower gastrointestinal recovery and gastrointestinal morbidity in patients undergoing bowel resection: pooled analysis of placebo data from 3 randomized controlled trials. Am J Surg 2006;191(3):315-9 [Epub 2006/02/24].

[11] Varadhan KK, Lobo DN. A meta-analysis of randomised controlled trials of intravenous fluid therapy in major elective open abdominal surgery: getting the balance right. Proc Nutr Soc 2010;69(4):488-98 [Epub 2010/06/03].

[12] Brandstrup B, Svendsen PE, Rasmussen M, et al. Which goal for fluid therapy during colorectal surgery is followed by the best outcome: near-maximal stroke volume or zero fluid balance? Br J Anaesth 2012;109(2):191 -9 [Epub 2012/06/20].

[13] Shires T, Williams J, Brown F. Acute change in extracellular fluids associated with major surgical procedures. Ann Surg 1961;154:803-10 [Epub 1961/11/01].

*[14] Chappell D, Jacob M, Hofmann-Kiefer K, et al. A rational approach to perioperative fluid management. Anesthesiology 2008;109(4):723-40 [Epub 2008/09/25].

[15] Lamke LO, Nilsson GE, Reithner HL. Water loss by evaporation from the abdominal cavity during surgery. Acta Chir Scand 1977;143(5):279-84 [Epub 1977/01/01].

[16] Miller TE, Roche AM, Gan TJ. Poor adoption of hemodynamic optimization during major surgery: are we practicing substandard care? Anesth Analg 2011;112(6):1274-6 [Epub 2011/05/27].

[17] Minto G, Struthers R. Stroke volume optimisation: is the fairy tale over? Anaesthesia 2014;69(4):291-6 [Epub 2014/03/ 20].

[18] Michard F. Stroke volume variation: from applied physiology to improved outcomes. Crit Care Med 2011;39(2):402-3 [Epub 2011/01/21].

[19] Marik PE, Cavallazzi R, Vasu T, et al. Dynamic changes in arterial waveform derived variables and fluid responsiveness in mechanically ventilated patients: a systematic review of the literature. Crit Care Med 2009;37(9):2642-7 [Epub 2009/07/ 16].

[20] Cecconi M, Monti G, Hamilton MA, et al. Efficacy of functional hemodynamic parameters in predicting fluid responsiveness with pulse power analysis in surgical patients. Minerva Anestesiol 2012;78(5):527-33 [Epub 2012/04/27].

[21] Le Manach Y, Hofer CK, Lehot JJ, et al. Can changes in arterial pressure be used to detect changes in cardiac output during volume expansion in the perioperative period? Anesthesiology 2012;117(6):1165-74 [Epub 2012/11/09].

[22] Marik PE, Baram M, Vahid B. Does central venous pressure predict fluid responsiveness? A systematic review of the literature and the tale of seven mares. Chest 2008;134(1):172-8 [Epub 2008/07/17].

[23] Renner J, Gruenewald M, Quaden R, et al. Influence of increased intra-abdominal pressure on fluid responsiveness predicted by pulse pressure variation and stroke volume variation in a porcine model. Crit Care Med 2009;37(2):650-8 [Epub 2008/12/31].

[24] Lansdorp B, Lemson J, van Putten MJ, et al. Dynamic indices do not predict volume responsiveness in routine clinical practice. Br J Anaesth 2012;108(3):395-401 [Epub 2011/12/22].

[25] Roche AM, Miller TE, Gan TJ. Goal-directed fluid management with trans-oesophageal Doppler. Best Pract Res Clin Anaesthesiol 2009;23(3):327-34 [Epub 2009/10/30].

[26] Gan TJ, Soppitt A, Maroof M, et al. Goal-directed intraoperative fluid administration reduces length of hospital stay after major surgery. Anesthesiology 2002;97(4):820-6.

[27] Noblett SE, Snowden CP, Shenton BK, et al. Randomized clinical trial assessing the effect of Doppler-optimized fluid management on outcome after elective colorectal resection. Br J Surg 2006;93(9):1069-76. 
[28] Wakeling HG, McFall MR, Jenkins CS, et al. Intraoperative oesophageal Doppler guided fluid management shortens postoperative hospital stay after major bowel surgery. Br J Anaesth 2005;95(5):634-42.

[29] Funk DJ, Moretti EW, Gan TJ. Minimally invasive cardiac output monitoring in the perioperative setting. Anesth Analg 2009;108(3):887-97 [Epub 2009/02/20].

[30] Goepfert MS, Reuter DA, Akyol D, et al. Goal-directed fluid management reduces vasopressor and catecholamine use in cardiac surgery patients. Intensive Care Med 2007;33(1):96-103 [Epub 2006/11/23].

[31] Pearse R, Dawson D, Fawcett J, et al. Early goal-directed therapy after major surgery reduces complications and duration of hospital stay. A randomised, controlled trial [ISRCTN38797445]. Crit Care 2005;9(6):R687-93 [Epub 2005/12/17].

[32] Benes J, Chytra I, Altmann P, et al. Intraoperative fluid optimization using stroke volume variation in high risk surgical patients: results of prospective randomized study. Crit Care 2010;14(3):R118 [Epub 2010/06/18].

[33] Cecconi M, Fasano N, Langiano N, et al. Goal-directed haemodynamic therapy during elective total hip arthroplasty under regional anaesthesia. Crit Care 2011;15(3):R132 [Epub 2011/06/01].

[34] Kapoor PM, Kakani M, Chowdhury U, et al. Early goal-directed therapy in moderate to high-risk cardiac surgery patients. Ann Cardiac Anaesth 2008;11(1):27-34 [Epub 2008/01/10].

[35] Lopes MR, Oliveira MA, Pereira VO, et al. Goal-directed fluid management based on pulse pressure variation monitoring during high-risk surgery: a pilot randomized controlled trial. Crit Care 2007;11(5):R100 [Epub 2007/09/08].

*[36] Cannesson M, Le Manach Y, Hofer CK, et al. Assessing the diagnostic accuracy of pulse pressure variations for the prediction of fluid responsiveness: a "gray zone" approach. Anesthesiology 2011;115(2):231-41 [Epub 2011/06/28].

[37] Muller L, Toumi M, Bousquet PJ, et al. An increase in aortic blood flow after an infusion of 100 ml colloid over 1 minute can predict fluid responsiveness: the mini-fluid challenge study. Anesthesiology 2011;115(3):541-7 [Epub 2011/07/28].

[38] Waldron NH, Miller TE, Thacker JK, et al. A prospective comparison of a noninvasive cardiac output monitor versus esophageal Doppler monitor for goal-directed fluid therapy in colorectal surgery patients. Anesth Analg 2014;118(5): $966-75$.

[39] Martina JR, Westerhof BE, van Goudoever J, et al. Noninvasive continuous arterial blood pressure monitoring with Nexfin(R). Anesthesiology 2012;116(5):1092-103.

[40] Bubenek-Turconi SI, Craciun M, Miclea I, et al. Noninvasive continuous cardiac output by the Nexfin before and after preload-modifying maneuvers: a comparison with intermittent thermodilution cardiac output. Anesth Analg 2013; 117(2):366-72.

[41] Chen G, Meng L, Alexander B, et al. Comparison of noninvasive cardiac output measurements using the Nexfin monitoring device and the esophageal Doppler. J Clin Anesth 2012;24(4):275-83.

*[42] Hamilton MA, Cecconi M, Rhodes A. A systematic review and meta-analysis on the use of preemptive hemodynamic intervention to improve postoperative outcomes in moderate and high-risk surgical patients. Anesth Analg 2011;112(6): 1392-402 [Epub 2010/10/23].

[43] Esophageal Doppler ultrasound-based cardiac output monitoring for real-time therapeutic management of hospitalized patients. Rochville: Agency for Healthcare Research and Quality, Department of Health \& Human Services; 2007.

[44] Ghosh S, Arthur B, Klein AA. NICE guidance on CardioQ(TM) oesophageal Doppler monitoring. Anaesthesia 2011;66(12): 1081-3 [Epub 2011/11/11].

*[45] Varadhan KK, Neal KR, Dejong CH, et al. The enhanced recovery after surgery (ERAS) pathway for patients undergoing major elective open colorectal surgery: a meta-analysis of randomized controlled trials. Clin Nutr 2010;29(4):434-40 [Epub 2010/02/02].

[46] Hausel J, Nygren J, Lagerkranser M, et al. A carbohydrate-rich drink reduces preoperative discomfort in elective surgery patients. Anesth Analg 2001;93(5):1344-50.

[47] Yuill KA, Richardson RA, Davidson HI, et al. The administration of an oral carbohydrate-containing fluid prior to major elective upper-gastrointestinal surgery preserves skeletal muscle mass postoperatively-a randomised clinical trial. Clin Nutr 2005;24(1):32-7.

[48] Wang ZG, Wang Q, Wang WJ, et al. Randomized clinical trial to compare the effects of preoperative oral carbohydrate versus placebo on insulin resistance after colorectal surgery. Br J Surg 2010;97(3):317-27.

[49] Srinivasa S, Taylor MH, Singh PP, et al. Randomized clinical trial of goal-directed fluid therapy within an enhanced recovery protocol for elective colectomy. Br J Surg 2013;100(1):66-74 [Epub 2012/11/08].

*[50] Pearse RM, Harrison DA, MacDonald N, et al. Effect of a perioperative, cardiac output-guided hemodynamic therapy algorithm on outcomes following major gastrointestinal surgery: a randomized clinical trial and systematic review. JAMA 2014;311(21):2181-90.

*[51] Mythen MG, Swart M, Acheson N, et al. Perioperative fluid management: consensus statement from the enhanced recovery partnership. Periop Med 2012;1(2):2.

[52] Grocott MP, Dushianthan A, Hamilton MA, et al. Perioperative increase in global blood flow to explicit defined goals and outcomes after surgery: a Cochrane Systematic Review. Br J Anaesth 2013;111(4):535-48 [Epub 2013/05/11].

[53] Finfer S, Liu B, Taylor C, et al. Resuscitation fluid use in critically ill adults: an international cross-sectional study in 391 intensive care units. Crit Care 2010;14(5):R185 [Epub 2010/10/19].

[54] Hadimioglu N, Saadawy I, Saglam T, et al. The effect of different crystalloid solutions on acid-base balance and early kidney function after kidney transplantation. Anesth Analg 2008;107(1):264-9 [Epub 2008/07/19].

[55] Wilkes NJ, Woolf R, Mutch M, et al. The effects of balanced versus saline-based hetastarch and crystalloid solutions on acidbase and electrolyte status and gastric mucosal perfusion in elderly surgical patients. Anesth Analg 2001;93(4):811-6.

[56] Scheingraber S, Rehm M, Sehmisch C, et al. Rapid saline infusion produces hyperchloremic acidosis in patients undergoing gynecologic surgery. Anesthesiology 1999;90(5):1265-70 [Epub 1999/05/13].

[57] Vogt NH, Bothner U, Lerch G, et al. Large-dose administration of 6\% hydroxyethyl starch 200/0.5 total hip arthroplasty: plasma homeostasis, hemostasis, and renal function compared to use of 5\% human albumin. Anesth Analg 1996;83(2): 262-8 [Epub 1996/08/01].

[58] Waters JH, Gottlieb A, Schoenwald P, et al. Normal saline versus lactated Ringer's solution for intraoperative fluid management in patients undergoing abdominal aortic aneurysm repair: an outcome study. Anesth Analg 2001;93(4): 817-22 [Epub 2001/09/28]. 
[59] Base EM, Standl T, Lassnigg A, et al. Efficacy and safety of hydroxyethyl starch 6\% 130/0.4 in a balanced electrolyte solution (Volulyte) during cardiac surgery. J Cardiothorac Vasc Anesth 2011;25(3):407-14 [Epub 2011/02/25].

[60] Shaw AD, Bagshaw SM, Goldstein SL, et al. Major complications, mortality, and resource utilization after open abdominal surgery: 0.9\% saline compared to Plasma-Lyte. Ann Surg 2012;255(5):821-9 [Epub 2012/04/04].

[61] Wilcox CS. Regulation of renal blood flow by plasma chloride. J Clin Invest 1983;71(3):726-35 [Epub 1983/03/01].

[62] Chowdhury AH, Cox EF, Francis ST, et al. A randomized, controlled, double-blind crossover study on the effects of 2-L infusions of $0.9 \%$ saline and plasma-lyte(R) 148 on renal blood flow velocity and renal cortical tissue perfusion in healthy volunteers. Ann Surg 2012;256(1):18-24 [Epub 2012/05/15].

*[63] Yunos NM, Bellomo R, Hegarty C, et al. Association between a chloride-liberal vs chloride-restrictive intravenous fluid administration strategy and kidney injury in critically ill adults. JAMA 2012;308(15):1566-72 [Epub 2012/10/18].

[64] Krebbel H, Feldheiser A, Muller O, et al. Influence of goal-directed therapy with balanced crystalloid-colloid or unbalanced crystalloid solution on base excess. J Int Med Res 2014;42(2):468-86 [Epub 2014/02/12].

[65] Myburgh J, Cooper J, Finfer S, et al. Saline or albumin for fluid resuscitation in patients with traumatic brain injury. N Engl J Med 2007;357(9):874-84.

[66] Cooper DJ, Myburgh J, Heritier S, et al. Albumin resuscitation for traumatic brain injury: is intracranial hypertension the cause of increased mortality? J Neurotrauma 2013;30(7):512-8.

[67] Jacob M, Chappell D, Hofmann-Kiefer K, et al. The intravascular volume effect of Ringer's lactate is below 20\%: a prospective study in humans. Crit Care 2012;16(3):R86 [Epub 2012/05/18].

*[68] Myburgh JA, Finfer S, Bellomo R, et al. Hydroxyethyl starch or saline for fluid resuscitation in intensive care. N Engl J Med 2012;367(20):1901-11.

[69] Perner A, Haase N, Guttormsen AB, et al. Hydroxyethyl starch 130/0.42 versus Ringer's acetate in severe sepsis. N Engl J Med 2012;367(2):124-34.

[70] Bihari S, Prakash S, Bersten AD. Post resusicitation fluid boluses in severe sepsis or septic shock: prevalence and efficacy (price study). Shock 2013;40(1):28-34 [Epub 2013/05/03].

*[71] Roger C, Muller L, Deras P, et al. Does the type of fluid affect rapidity of shock reversal in an anaesthetized-piglet model of near-fatal controlled haemorrhage? A randomized study. Br J Anaesth 2014;112:1015-23 [Epub 2013/12/03].

[72] Yates DR, Davies SJ, Milner HE, et al. Crystalloid or colloid for goal-directed fluid therapy in colorectal surgery. Br J Anaesth 2014;112(2):281-9 [Epub 2013/09/24].

[73] Feldheiser A, Pavlova V, Bonomo T, et al. Balanced crystalloid compared with balanced colloid solution using a goaldirected haemodynamic algorithm. Br J Anaesth 2013;110(2):231-40 [Epub 2012/11/01].

[74] Holte K, Klarskov B, Christensen DS, et al. Liberal versus restrictive fluid administration to improve recovery after laparoscopic cholecystectomy: a randomized, double-blind study. Ann Surg 2004;240(5):892-9 [Epub 2004/10/20].

[75] Lambert KG, Wakim JH, Lambert NE. Preoperative fluid bolus and reduction of postoperative nausea and vomiting in patients undergoing laparoscopic gynecologic surgery. AANA J 2009;77(2):110-4 [Epub 2009/04/25].

[76] Maharaj CH, Kallam SR, Malik A, et al. Preoperative intravenous fluid therapy decreases postoperative nausea and pain in high risk patients. Anesth Analg 2005;100(3):675-82. table of contents. [Epub 2005/02/25].

[77] Miller TE, Thacker JK, White WD, et al. Reduced length of hospital stay in colorectal surgery after implementation of an enhanced recovery protocol. Anesth Analg 2014;118(5):1052-61.

[78] Annane D, Siami S, Jaber S, et al. Effects of fluid resuscitation with colloids vs crystalloids on mortality in critically ill patients presenting with hypovolemic shock: the CRISTAL randomized trial. JAMA 2013;310:1809-17. 